MMCA-97 Conference, pp. 75-78

R. Čiegis (Ed)

(C) 1997 Vilniaus Gedimino technikos universitetas

\title{
THE NONLINEAR CONVOLUTIONAL EQUATIONS SOLVABLE IN CLOSED FORM
}

\author{
V.A. KAKICHEV \\ Novgorod State University, Dept. of Theoretical Physics \\ Novgorod, 173003, RUSSIA. \\ E-mail: teorfis@lan.novsu.ac.ru
}

\section{SECTION 1}

Let C: $H \leftrightarrow W$ is bijective linear operator, operating from algebra $W$ in linear space $\mathrm{H}$. We define convolution with weight $\gamma \in W$ by equation $[1,2]$

$$
\varphi * \psi=C^{-1}[\gamma \cdot(C \varphi) \cdot(C \psi)], \varphi, \psi \text { and } \varphi * \psi \in H
$$

Then

$$
\begin{gathered}
\varphi * \psi=\psi * \varphi \text { and }(\varphi * \psi) * \chi=\varphi *(\psi * \chi), \forall \varphi, \psi, \chi \in H \\
(\varphi * \psi) * \chi=C^{-1}\{\gamma \cdot[C(\varphi * \psi)] \cdot(C \chi)\}=C^{-1}\left[\gamma^{2} \cdot C(\varphi) \cdot C(\psi) \cdot(C \chi)\right]= \\
\varphi *(\psi * \chi), \varphi, \psi, \chi \in H
\end{gathered}
$$

Then from here the possibility of definition of "power" of convolution follows $[3,4]$

$$
(\varphi)^{n}=\underbrace{\varphi * \varphi * \ldots * \varphi}_{n}=C^{-1}\left[\gamma^{n-1} \cdot C(\varphi)^{n}\right], n \in N .
$$

Particular cases are:

$$
(\varphi)^{1}=\varphi,(\varphi)^{n} *(\varphi)^{m}=(\varphi)^{n+m} \forall n, m \in N .
$$

We shall suppose further, that the algebra $H$ is commutative normalized ring [5] concerning of norm $\|\cdot\|$ such, that

$$
\|\varphi * \psi\| \leq\|\varphi\| \cdot\|\psi\|, \forall \varphi, \psi \in H \text { and, particularly, }\left\|(\varphi)^{n}\right\| \leq\|\varphi\|^{n}
$$

At last, we shall suppose, that for element $\varphi \in H$ exists finite or infinite limit

$$
\lim _{n \rightarrow \infty}\left[\gamma^{n-1} \cdot(C \varphi)^{n}\right]=\varphi_{\infty}
$$

where from $\lim _{n \rightarrow \infty}(\varphi)^{n}=C^{-1}\left(\varphi_{\infty}\right)$ or belong to $H$, or this limit is infinite. 


\section{SECTION 2}

Assuming, that $\gamma^{-1} \in W$, power series has form (3)

$$
y=a(x)=\sum_{n=1}^{\infty} a_{n} x^{n}, a_{1}=1,|x|<r_{a} .
$$

and we compare "convolutional" series (compare [4]) to power series (3)

$$
\begin{array}{r}
\sum_{n=1}^{\infty} a_{n}(\varphi)^{n}=\sum_{n=1}^{\infty} a_{n} C^{-1}\left[\gamma^{n-1} \cdot C(\varphi)^{n}\right]=C^{-1}\left[\sum_{n=1}^{\infty} a_{n} \gamma^{n-1} \cdot C(\varphi)^{n}\right]= \\
C^{-1}\left\{\gamma^{-1} \cdot \sum_{n=1}^{\infty} a_{n}\left[\gamma^{n} \cdot C(\varphi)^{n}\right]\right\}=C^{-1}\left\{\gamma^{-1} \cdot a\left[\gamma \cdot C(\varphi)^{n}\right]\right\}
\end{array}
$$

which, obviously, make sence, if $\|\varphi\| \leq r_{a}$.

Let the series

$$
x=\sum_{k=1}^{\infty} b_{k} y^{k}, b_{1}=1,|y|<r_{b}
$$

convert series (3):

$$
b(a(x))=x,|x|<s_{a}<r_{a} a(b(y))=y,|y|<s_{b}<r_{b},
$$

then the factors $b_{k}$ of series (5) determining (see p.755 in [6]) by formulae:

$$
\begin{aligned}
& b_{m}=\sum_{\alpha_{2}+2 \alpha_{3}+\cdots+(m-1) \alpha_{m}=m-1} \frac{\left(m-1+\alpha_{2}+\cdots+\alpha_{m}\right) !}{m ! \alpha_{2} ! \cdots \alpha_{m} !} \\
& \times\left(-a_{2}\right)^{\alpha_{2}} \cdot \ldots \cdot\left(-a_{m}\right)^{\alpha_{m}},
\end{aligned}
$$

where $\alpha_{j} \in N$.

In particular, if series (3) is polinomial of power $p$, then instead (7) we have:

$$
b_{m}=\sum_{\alpha_{2}+2 \alpha_{3}+\cdots+(p-1) \alpha_{p}=m-1} \frac{\left(m-1+\alpha_{2}+\cdots+\alpha_{p}\right) !}{m ! \alpha_{2} ! \ldots \alpha_{p} !} \cdot\left(-a_{2}\right)^{\alpha_{2}} \cdot \ldots \cdot\left(-a_{p}\right)^{\alpha_{p}} .
$$




\section{SECTION 3}

We consider convolutional equation

$$
a(\varphi)=\sum_{n=1}^{\infty} a_{n}(\varphi)^{n}=\psi
$$

in which $\psi$ - is known, and $\varphi$ - is required element from $\mathrm{H}$. In general case this equation have infinite order, but it may be polynomial equation of power $p \geq 2$.

We allows, that function $a(x)$ is invertible with help series (5) and, hence, the equality (6) and (7) (or (8)) take place. We notice, that function $a(x)$, is strictly monotonic on interval $\left\{|x|<s_{a}<r_{b}\right\}$, is obviously invertible.

Takinig into account (4) we find that $b(\gamma \cdot(C \psi))=\gamma \cdot(C \varphi)$ or

$$
\varphi=C^{-1}\left[\gamma^{-1} \cdot b(\gamma \cdot(C \psi))\right]=\sum_{n=1}^{\infty} b_{n}(\psi)^{n}
$$

From here, if $\|\psi\|<s_{b}$, then equality (9) has the solution $\varphi \in H$, determined by formula (10) $\|\varphi\|<s_{a}$ and by virtue of (1).

It is easy to see, that convolutional equation

$$
\sum_{n=1}^{\infty} b_{n}(\varphi)^{n}=\psi, \psi \in H,\|\psi\|<s_{b}
$$

has the solution

$$
\varphi=\sum_{n=1}^{\infty} a_{n}(\psi)^{n} \in H,\|\varphi\|<s_{a}
$$

\section{SECTION 4}

We made two remarks:

a) Departing from decomposition of kind [7]

$$
\begin{gathered}
\sin \lambda x=2 \sum_{n=1}^{\infty}(-1)^{n+1} J_{2 n-1}(\lambda) T_{2 n-1}(x)= \\
\frac{2}{\lambda} \sum_{n=1}^{\infty}(-1)^{n} J_{2 n}(\lambda) U_{2 n-1}(x),|x|<1,0<\lambda<\pi
\end{gathered}
$$

naturally series (3) to replace on more general series

$$
\sum_{n=1}^{\infty} b_{n} Q_{2 n-1}(x), Q_{2 n-1}(x)=\sum_{s=1}^{n} q_{n, s} x^{2 s-1} .
$$

b) Probably, for the first time particular nonlinear equation in convolutions with $\mathrm{p}=2$ was investigated in article [8]. One of variants of research of polynomial equations with convolutions is offered in [4]. 


\section{REFERENCES}

[1] V.A. Kakichev, About convolutions for integral transformations. Izv. Belorussian SSR.- Ser.: fiz-mat., 1967, No 2, P. 48-57. (In Russian).

[2] Kakichev V.A, About matrix convolution of power series. Izv. vuzov: Matem., 1990, No 2, P. 53-62. (In Russian).

[3] Kakichev V.A. Trigonometric convolutions as the means summation of series. Uch.zap.MOPI.V.269.Mat.analiz.V.14-M.;, 1969, P. 144-165. (In Russian).

[4] Yakubovich S.B., Luchko Yu.F. The operational properties of convolution for Kantorovich-Lebedev transformations. Dokl. AN Belorussii, 1994, 38(4), P.19-23. (In Russian).

[5] Gelfand I.M., Raikov D.A., Shilov G.E. Commutative normalized rings. M.:Fizmatgiz, 1960, 316 p. (In Russian).

[6] Prudnikov A.P., Bruchkov Yu.A., Maruchev O.H. The integrals and series. M.: Nauka, V.3, , 1986. (In Russian).

[7] Lusternik L.A., Chernonenkis O.A., Yampolskii A.P. Mathematical Analises. Calculation of elementary functions. M.: Fizmatgiz., 1963, 246 p. (In Russian).

[8] Saveliev G.I. About one nonlinear convolutional equation. Trudy. Polytekhn. in-ta., Novocherkassk, 1959, No 100, P. 47-52. (In Russian). 